\title{
SIMULAÇÃO NUMÉRICA E FÍSICA NA SOLDAGEM DOS AÇOS INOXIDÁVEIS DUPLEX: UMA REVISÃO SISTEMÁTICA.
}

Leonardo Oliveira Passos da Silva1; Advan Coelho Nascimento'; Francisco Magalhães dos Santos Júnior ${ }^{1}$, Tiago Nunes Limaํ․, Bruno Caetano dos Santos Silva ${ }^{1}$, Rodrigo Santiago Coelho ${ }^{1}$

${ }^{1}$ SENAI CIMATEC, Av. Orlando Gomes, 1845 - Piatã, 41650-010; Salvador/Bahia; leooliveirap@gmail.com

Resumo: A soldagem é um processo muito utilizado em diversos segmentos industriais, que necessita de controle dos seus parâmetros, especialmente quando se trata da soldagem de aços inoxidáveis duplex (AID). Este trabalho apresenta uma revisão sistemática sobre a utilização combinada de simulações numéricas e físicas para predição do comportamento de um AID durante a soldagem. A pesquisa foi realizada tendo como base o banco de dados da plataforma ScienceDirect. Os resultados apresentaram apenas três artigos sobre simulações numéricas e físicas para a soldagem de AID. Percebe-se, também, um crescente interesse nesse tema, uma vez que $50 \%$ dos trabalhos foram publicados nos últimos 5 anos e ressaltam que as simulações podem ser usadas para predizer este processo.

Palavras-Chave: Revisão sistemática; Soldagem; Aço inoxidável duplex; Simulação Numérica; Gleeble ${ }^{\circledR}$.

\section{NUMERICAL AND PHYSICAL SIMULATION FOR DUPLEX STAINLESS STEEL WELDING: A SYSTEMATIC REVIEW.}

\begin{abstract}
The welding process stands out for being widely used in manufacturing, that it requires control of its parameters, especially when welding duplex stainless steels (DSS). This paper presents a systematic review of the literature on the use of numerical and physical simulations to predict the behavior of a DSS during welding. The database used was articles published on the ScienceDirect ${ }^{\circledR}$ platform. The results show the low number of studies regarding numerical and thermomechanical simulations for welding duplex stainless steels. There is a growing interest in this topic, insofar as $50 \%$ of papers have been published in the last five years. The researched literature emphasized that simulations can be used to predict the welding process.
\end{abstract}

Keywords: Systematic review; Welding; Duplex stainless steel; Numerical simulation; Gleeble ${ }^{\circledR}$. 


\section{INTRODUÇÃO}

O desenvolvimento e a aplicação de novas tecnologias, relacionados à manufatura, têm como objetivo proporcionar melhor desempenho operacional com foco em produtividade e qualidade. A manufatura é composta, dentre outros, pelos processos de conformação, usinagem e soldagem [1]. Este último, destaca-se devido, principalmente, pela sua flexibilidade e vasta aplicação industrial [2-4].

Para aplicações de alta responsabilidade, destaca-se a utilização dos aços inoxidáveis duplex, principalmente na indústria química, petroquímica, e, mais recentemente, na indústria offshore [5-8]. Isto se deve por combinarem excelentes propriedades mecânicas e corrosivas obtidos pelo balanço microestrutural aproximadamente igual entre a ferrita e a austenita [5]. Quando comparados aos inoxidáveis austeníticos, os AID's são de menor custo devido ao menor teor de $\mathrm{Ni}[5,6]$.

Nas estruturas soldadas é comum a existência de tensões residuais causadas, principalmente, pela expansão e contração térmica não uniforme $[4,9,10]$. Durante o processo de soldagem dos AID's também é passível de ocorrer a precipitação de fases deletérias, que afetam a integridade das juntas soldadas, aumentando a suscetibilidade à fadiga, corrosão sob tensão e fraturas frágeis [4]. A formação dessas fases como, por exemplo, as fases sigma, chi, carbetos e nitretos, pode levar a falha prematura dos componentes [11].

Apesar das excelentes propriedades dos AID's, predizer as tensões residuais e a ocorrência das fases deletérias é um desafio crucial para garantir o desempenho adequado em operação, as quais perpassam pela necessidade de controlar o aporte térmico e taxa de resfriamento durante a soldagem [12-14]. Baixo aporte térmico e, consequente alta taxa de resfriamento, interferem no balanço microestrutural, diminuindo a fração volumétrica da austenita e, assim, a resistência corrosiva. Por outro lado, alto aporte térmico, e baixa taxa de resfriamento, permitem a precipitação de fases intermetálicas com consequente fragilização do aço [12-14].

As ferramentas de simulação física e numérica vêm mostrando um potencial enorme para auxiliar os processos de soldagem e apresentam uma utilização cada vez maior, apesar de ainda possuírem uma utilização pouco representativa e difundida nas empresas do setor $[13,14]$. A simulação da zona termicamente afetada (ZTA) é capaz de reproduzir os ciclos térmicos a partir da escolha de modelos de fluxo de calor. O ciclo térmico pode ser obtido por meio de soldas reais registrados por termopares, ou através da aquisição de dados gerados por um simulador computacional como, por exemplo, o Simufact Welding [15-17].

A etapa mais crítica em uma simulação de soldagem é a escolha dos parâmetros de trabalho e das condições de contorno. Estes parâmetros devem ser compatíveis com a solda real e necessitam garantir repetibilidade e qualidade em um processo de simulação [14-17]. No contexto atual da manufatura digital sustentada pela Indústria 4.0, o uso de simulações se tornou essencial na virtualização da produção $[15,16]$. O emprego de simuladores termomecânicos, como a Gleeble ${ }^{\circledR}$, e numéricos em experimentos, tem se tornado essenciais para a otimização dos processos de soldagem $[17,18]$. 
A Figura 1 evidencia a metodologia do desenvolvimento da digitalização do processo de soldagem. Com o material e processo selecionados, esta sistemática visa utilizar a simulação numérica para a definição precisa de parâmetros de soldagem, como as temperaturas do ciclo térmico, e investigar a resposta no simulador termomecânico. Após criado o procedimento, validar a confiabilidade dos modelos com testes laboratoriais e em soldas reais [18].

Figura 1. Esquemático da metodologia a ser explorada.

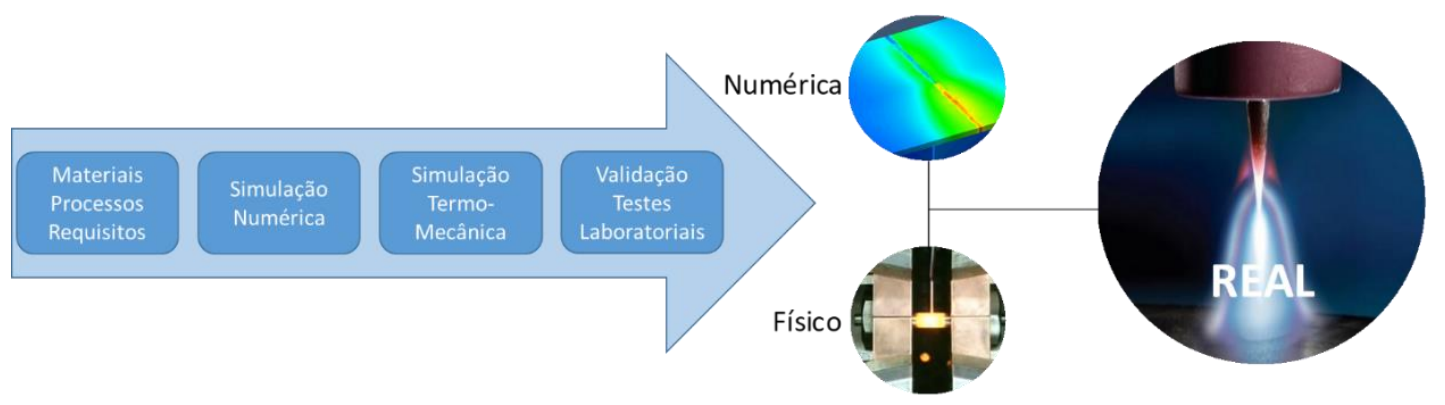

Em face ao apresentado, o presente trabalho tem como objetivo realizar uma revisão sistemática do estado da arte sobre o uso de simulações numéricas e físicas (usando a Gleeble ${ }^{\circledR}$ ), em um aço inoxidável duplex durante a sua soldagem.

\section{METODOLOGIA}

A metodologia consistiu no levantamento de publicações e utilização da sistemática descrita a seguir:

I. Elaboração de perguntas norteadoras, vide Tabela 1;

Tabela 1. Questões norteadoras.

\begin{tabular}{c|l}
$\#$ & \multicolumn{1}{|c}{ Questões norteadoras } \\
$\mathbf{1}$ & $\begin{array}{l}\text { Existem trabalhos que utilizam simultaneamente: processo de soldagem, aço } \\
\text { inoxidável duplex e simulações? }\end{array}$ \\
\hline $\mathbf{2}$ & $\begin{array}{l}\text { Existem trabalhos em que a resposta da simulação numérica foi utilizada } \\
\text { como input para a simulação física com a Gleeble }{ }^{\circledR} ?\end{array}$ \\
\hline $\mathbf{3}$ & $\begin{array}{l}\text { Existem trabalhos em que a simulação numérica e física foram validadas em } \\
\text { soldas reais? }\end{array}$ \\
\hline
\end{tabular}

II. Seleção das plataformas eletrônicas de busca de periódicos acadêmicos;

III. Definição de descritores e combinações com busca de palavras-chave de acordo com a plataforma selecionada, conforme Tabela 2; 


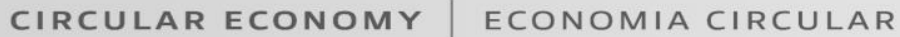

Tabela 2. Relação das palavras-chave utilizadas na plataforma ScienceDirect.

\begin{tabular}{c|l}
$\#$ & \multicolumn{1}{c}{ Palavras-chave } \\
\hline $\mathbf{1}$ & Welding \\
\hline $\mathbf{2}$ & (Welding OR weld) AND stainless steel \\
\hline $\mathbf{3}$ & (Welding OR weld) AND duplex \\
\hline $\mathbf{4}$ & $\begin{array}{l}\text { (Welding OR weld) AND (duplex) AND (finite element OR simufact welding OR } \\
\text { abaqus OR ansys OR numerical simulation) }\end{array}$ \\
\hline $\mathbf{5}$ & $\begin{array}{l}\text { (Welding OR weld) AND (duplex) AND (finite element OR simufact welding OR } \\
\text { abaqus OR ansys OR numerical simulation) AND gleeble }\end{array}$ \\
\hline
\end{tabular}

IV. Seleção primária dos artigos encontrados de acordo com critérios de inclusão, apresentados na Tabela 3;

Tabela 3. Critérios de inclusão.

\begin{tabular}{c|l}
\multicolumn{1}{c}{ \# } & \multicolumn{1}{c}{ Critérios } \\
$\mathbf{1}$ & Artigos acadêmicos referente a soldagem de aços inoxidáveis duplex; \\
\hline $\mathbf{2}$ & $\begin{array}{l}\text { Artigos acadêmicos que relacionam a soldagem de aços inoxidáveis duplex } \\
\text { com ferramentas de simulação; }\end{array}$ \\
\hline $\mathbf{3}$ & Artigos na língua inglesa; \\
\hline $\mathbf{4}$ & Artigos disponibilizados na íntegra; \\
\hline $\mathbf{5}$ & Acesso gratuito aos artigos. \\
\hline
\end{tabular}

V. Seleção secundária de trabalhos após leitura integral do artigo;

VI. Análise quantitativa dos dados coletados;

VII. Análise qualitativa, com identificação dos trabalhos que respondem às perguntas norteadoras.

\section{RESULTADOS E DISCUSSÃO}

\subsection{Análise quantitativa dos dados}

A Figura 2 exibe um gráfico comparativo, em escala logarítmica, da pesquisa de publicações científicas utilizando o resultado das combinações apresentadas na Tabela 2, do quantitativo global encontrado. O gráfico evidencia o afunilamento das pesquisas e a tendência de crescimento no interesse dos temas propostos nos últimos cinco anos. Esta informação está em destaque, apresentada na forma do número absoluto e em porcentagem. 
Figura 2. Comparação do número de publicações nas combinações pesquisadas de 1 a 5 .

Em destaque, o total e a porcentagem dos últimos 5 anos. Base ScienceDirect

(www.sciencedirect.com). Dados coletados em 05/08/2019 às 19:30.

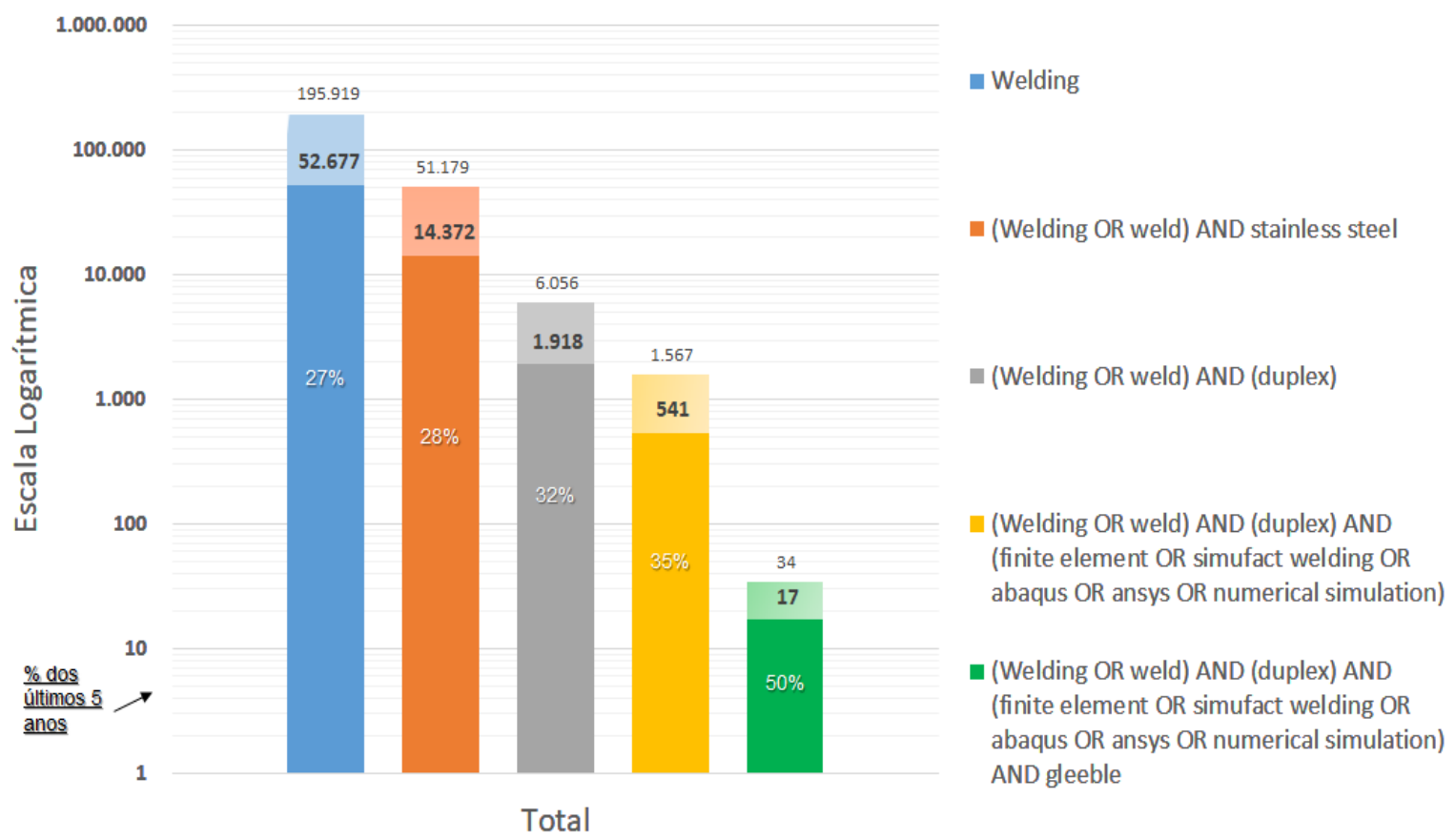

A partir dos resultados obtidos é possível inferir a relevância dos aços inoxidáveis e sua relação com o processo de soldagem, sendo 51.179 do total de publicações sobre soldagem usou o aço inoxidável como material de pesquisa. A análise mostra também que, dentre o universo de 195.919 trabalhos publicados sobre soldagem, 1.567 envolvem algum grau de uso da simulação numérica e apenas 34 encontrados com as palavras-chaves que envolvem soldagem, simulação numérica e o equipamento para simulação termomecânica (Gleeble ${ }^{\circledR}$ ). Deste último grupo, 50\% (17) foram publicados nos últimos cinco anos.

De acordo com a metodologia utilizada, o levantamento bibliográfico da combinação 5 da Tabela 2 encontrou ao todo 34 resultados. Após a aplicação dos critérios de inclusão, 14 foram excluídos. Após a leitura na íntegra, 17 artigos foram excluídos por um dos seguintes motivos: não se tratar do processo de soldagem; não utilizar o material duplex; não usar softwares de simulação nos estudos ou ambos motivos, restando apenas três. A Figura 3 apresenta o fluxograma das etapas de identificação, seleção, elegibilidade e inclusão dos artigos.

Figura 3. Fluxograma da seleção de artigos para revisão sistemática.

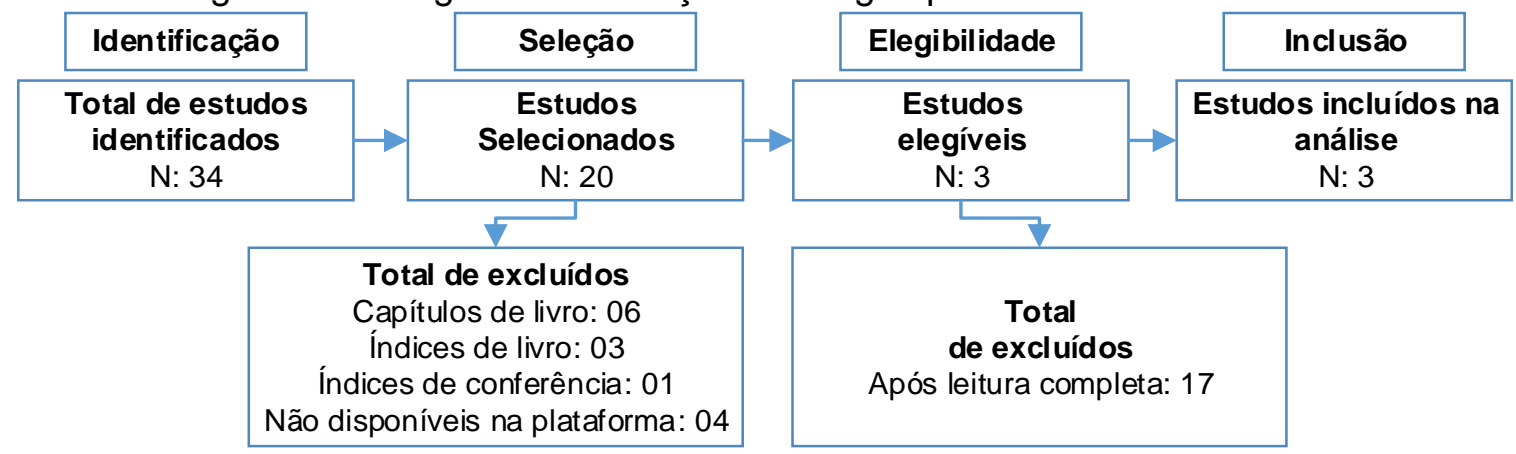




\subsection{Análise qualitativa dos dados}

O estudo de GARZÓN, C. M.; RAMIRES, A. J., 2006 [19], analisou o efeito de diferentes ciclos térmicos na formação de austenita secundária na Zona Termicamente Afetada (ZTA) do aço inoxidável UNS S32304. Este material foi submetido a ciclos térmicos na Gleeble ${ }^{\circledR}$ e analisado nos microscópios ótico, eletrônica de varredura e de transmissão. Os softwares de simulação numérica, ThermoCalc $^{\circledR}$ e Módulo DICTRA ${ }^{\circledR}$, forneceram a avaliação termodinâmica e cinética do crescimento da austenita, dissolução de nitretos e precipitados. Os autores concluíram que os resultados das simulações numéricas foram bastante similares com a caracterização microestrutural após a simulação com a Gleeble ${ }^{\circledR}$. Apesar de correlacionar os resultados da simulação física e numérica, o artigo não respondeu integralmente às perguntas norteadoras pois não utilizou a soldagem real para validação dos resultados.

Já HOSSEINI, V. A; et. al., 2017 [20], abordaram uma outra técnica de simulação termomecânica da ZTA ao invés da Gleeble ${ }^{\circledR}$. Para isso, foi utilizada uma tocha do processo GTAW como fonte de calor e uma amostra do aço superduplex SAF 2507 para os testes. Esta técnica reproduziu gradientes das temperaturas na soldagem com o objetivo de reduzir o número de amostras necessárias para caracterizar a resposta do material à soldagem. A simulação numérica foi utilizada para calcular o perfil de distribuição térmica na amostra, com os softwares JMatPro ${ }^{\circledR}$ e OpenFOAM ${ }^{\circledR}$, obtendo, assim, as propriedades termodinâmicas, diagrama de fase e a curva tempo temperatura e transformação (curva TTT) para aquisição dos parâmetros e inseri-los na simulação física. Os autores não utilizaram a Gleeble ${ }^{\circledR}$ para a simulação física, mas sim uma proposta de novo modelo calibrado com a simulação numérica. Similar com GARZÓN, C. M.; RAMIRES, A. J., 2006, o estudo de HOSSEINI, V. A; et. al., 2017, não confrontaram os resultados com uma solda real.

Em seguida, HOSSEINI, V. A; KARLSSON, L., 2019 [21], prosseguiram os estudos referentes ao processo GTAW no material SAF 2507, com a análise da perda do nitrogênio na ZTA de alta temperatura. O estudo incluiu simulação física, utilizando a técnica apresentada no estudo anterior e a Gleeble ${ }^{\circledR}$, soldagem real com o processo GTAW e simulação numérica com os softwares ThermoCalc ${ }^{\circledR}$ e DICTRA $^{\circledR}$. Os resultados encontrados mostraram que houve perda do nitrogênio da ZTA de alta temperatura para a poça de fusão. Esta perda foi apontada pela simulação numérica, confirmada pela simulação física e em concordância com os resultados da soldagem real. $\mathrm{O}$ uso combinado das simulações também previu adequadamente a fração da fase ferrítica e os experimentos físicos e soldagem GTAW. Esta abordagem permitiu comparar o resultado da soldagem real com a numérica e física. Este artigo, dentre os analisados, foi o único que respondeu integralmente as perguntas norteadoras, pois neste estudo foi utilizado softwares de simulações numéricas e a Gleeble ${ }^{\circledR} \mathrm{com}$ o propósito de prever o comportamento do material em uma solda real. 


\section{CONCLUSÃO}

Neste artigo foi realizada uma revisão sistemática da literatura com o objetivo de mapear publicações que tratam sobre o uso de simulação numérica e física para predizer o comportamento de soldagem dos aços inoxidáveis duplex. Do universo de 195.919 artigos com o tema soldagem na plataforma do ScienceDirect, trinta e quatro foram detectados utilizando as palavras-chave selecionadas. Destes, apenas um artigo respondeu integralmente as perguntas norteadoras.

A literatura estudada ressaltou que a utilização combinada das simulações numéricas e simulações físicas na Gleeble ${ }^{\circledR}$ pode ser usada para predizer 0 comportamento do material no processo de soldagem com precisão. E percebe-se, também, o número escasso de trabalhos sobre a aplicação de simulações numéricas e físicas com a Gleeble ${ }^{\circledR}$ para predizer o comportamento de AID’s na soldagem, mas que há um interesse crescente neste tema, principalmente nos últimos cinco anos.

\section{REFERÊNCIAS}

1 SAOUBI, R.M.; CHANDRASEKARAN, H. Role of phase and grain size on chip formation and material work hardening during machining of single and dual phase steels. Ironmaking and Steelmaking, v. 31, n. 3, p. 258-264, 2014.

${ }^{2}$ GUERRA, I.M. Soldagem \& Técnicas Conexas: Processos. Porto Alegre, 2007.

${ }^{3}$ MARQUES P.V.; MODENESE P.J.; BRACARENSE A.Q. Soldagem Fundamentos e Tecnologia. 3 ${ }^{\underline{a}}$ edição. Belo Horizonte, 2011.

${ }^{4}$ LEE, C.H.; CHANG, K.H. Comparative study on girth weld-induced residual stresses between austenitic and duplex stainless steel pipe welds. Applied Thermal Engineering, n. 63, p. 140-150, 2014.

${ }^{5}$ SILVA, A.L.V.C.; MEI, P.R. Aços e ligas especiais. 2ª edição, São Paulo: Blucher, 2006.

${ }^{6}$ ZHANG, Z.; et. al. Influence of microstructure and elemental partitioning on pitting corrosion resistance of duplex stainless steel welding joints. Applied Surface Science, n. 394, p. 297-314, 2017.

7 YANG, J.; et. al. Weld failure analysis of 2205 duplex stainless steel nozzle. Case Studies in Engineering Failure Analysis, n. 2, p. 69-75, 2014.

${ }^{8}$ SANTOS, D.C.; MAGNABOSCO, R. Kinetic study to predict sigma phase formation in duplex stainless steels. Metallurgical and Materials Transactions A, v. 47A, p. 1554-1565, 2016.

${ }^{9}$ RAVISANKAR, A.; et al. Influence of welding speed and power on residual stress during gas tungsten arc welding (GTAW) of thin sections with constant heat input: $A$ study using numerical simulation. Journal of Manufacturing Processes, n. 16, p. 200-211, 2014. 
${ }^{10}$ PUYMBROECK, E.V.; et al. Residual weld stresses in stiffener-to-deck plate weld of an orthotropic steel deck. Journal of Constructional Steel Research, n. 159, p. 534$547,2019$.

11 ARUN, D.; et al. Multi-pass arc welding techniques of $12 \mathrm{~mm}$ thick super-duplex stainless steel. Journal of Materials Processing Tech., n. 271, p. 126-143, 2019.

12 JEON, J.T.; et al. Behavior of a girth-welded duplex stainless steel pipe under external pressure. Ocean Engineering, n. 109, p. 93-102, 2015.

13 LUNDIN, C. D.; ZHOU, G. A comparison of published HAZ thermal simulation methods used to derive weld haz thermal cycles. Acta Metallurgica Sinica, v. 13, n. 1, p. 223-232, 2000.

${ }^{14}$ ANDONYI, Y. Heat-affected zone characterization by physical simulations. Welding Journal, 2006, p. 42-47.

15 CHRYSSOLOURIS, G.; et. al. Digital manufacturing: history, perspectives, and outlook. Proc. IMechE, v. 223 Part B: J. Engineering Manufacture, 2009.

${ }^{16}$ NOF, S.Y.; SILVA, J.R. Perspectives on manufacturing automation under the digital and cyber convergence. Polytechnica, 2018.

17 JABBARI, M.; et. al. Multiphysics modelling of manufacturing processes: A review. Advances in Mechanical Engineering, v. 10(5), p. 1-31, 2018.

${ }^{18}$ COELHO, R.S.; et. al. Digital manufacturing: challenges and perspectives for the materials forming \& joining industry in Brazil. In: 38 SENAFOR, Brasil, 2018.

19 GARZÓN, C.M.; RAMIRES, A.J. Growth kinetics of secondary austenite in the welding microstructure of a UNS S32304 duplex stainless steel. Acta Materialia, n. 54, p. 3321-3331, 2006.

${ }^{20}$ HOSSEINI, V.A; et. al. A novel arc heat treatment technique for producing graded microstructures through controlled temperature gradients. Materials and Design, $\mathrm{n}$. 121, p. 11-23, 2017.

${ }^{21}$ HOSSEINI, V.A; KARLSSON, L. Physical and kinetic simulation of nitrogen loss in high temperature heat affected zone of duplex stainless steels. Materialia, n. 6, p. 10325, 2019. 